\title{
Detailed Cache Coherence Characterization for OpenMP Benchmarks .
}

\author{
Jaydeep Marathe ${ }^{1}$, Anita Nagarajan², Frank Mueller ${ }^{1}$ \\ ${ }^{1}$ Dept. of Computer Science, North Carolina State University, Raleigh, NC 27695-7534 \\ 2 Intel Technology India Pvt. Ltd., 136, Airport Road, Bangalore - 560017, India \\ mueller@cs.ncsu.edu, phone: +1.919.515.7889, fax: +1.919.515.7925
}

\begin{abstract}
Past work on studying cache coherence in shared-memory symmetric multiprocessors (SMPs) concentrates on studying aggregate events, often from an architecture point of view. However, this approach provides insufficient information about the exact sources of inefficiencies in parallel applications. For SMPs in contemporary clusters, application performance is impacted by the pattern of shared memory usage, and it becomes essential to understand coherence behavior in terms of the application program constructs such as data structures and source code lines.

The technical contributions of this work are as follows. We introduce ccSIM, a cache-coherent memory simulator fed by data traces obtained through on-the-fly dynamic binary rewriting of OpenMP benchmarks executing on a Power3 SMP node. We explore the degrees of freedom in interleaving data traces from the different processors and assess the simulation accuracy by comparing with hardware performance counters. The novelty of ccSIM lies in its ability to relate coherence traffic - specifically coherence misses as well as their progenitor invalidations - to data structures and to their reference locations in the source program, thereby facilitating the detection of inefficiencies. Our experiments demonstrate that (a) cache coherence traffic is simulated accurately for SPMD programming styles as its invalidation traffic closely matches the corresponding hardware performance counters, (b) we derive detailed coherence information indicating the location of invalidations in the application code, i.e, source line and data structures and (c) we illustrate opportunities for optimizations from these details. By exploiting these unique features of ccSIM, we were able to identify and locate opportunities for program transformations, including interactions with OpenMP constructs, resulting in both significantly decreased coherence misses and savings of up to $73 \%$ in wall-clock execution time for several real-world benchmarks.
\end{abstract}

\section{Categories and Subject Descriptors}

D.1.3 [Concurrent Programming]: Parallel Programming

\begin{abstract}
${ }^{*}$ This work was supported in part by NSF CAREER grant CCR0237570 and through the U.S. Department of Energy by the University of California, Lawrence Livermore National Laboratory under subcontract \# B540203.
\end{abstract}

Permission to make digital or hard copies of all or part of this work for personal or classroom use is granted without fee provided that copies are not made or distributed for profi t or commercial advantage and that copies bear this notice and the full citation on the fi rst page. To copy otherwise, to republish, to post on servers or to redistribute to lists, requires prior specifi c permission and/or a fee.

ICS'04, June 26-July 1, 2004, Saint-Malo, France.

Copyright 2004 ACM 1-58113-839-3/04/0006 ...\$5.00

\section{General Terms}

Measurement, Performance

\section{Keywords}

Dynamic binary rewriting, program instrumentation, cache analysis, SMPs, coherence protocols

\section{Introduction}

Prior work on cache coherence concentrates on two areas: simulation and performance tuning. Many architectural and system simulators support different coherence models in their implementation (e.g., $[7,14,27,25,2,8])$, and they operate at different abstraction levels ranging from cycle-accuracy over instruction-level to the operating system interface. On the performance tuning end, work mostly concentrates on program analysis to derive optimized code (e.g., $[16,28])$. Recent processor support for performance counters opens new opportunities to study the effect of applications on architectures with the potential to complement them with per-reference statistics obtained by simulation.

In this paper, we focus on cache coherence simulation without cycle accuracy or even instruction-level simulation. We constrain ourselves to an SPMD programming paradigm on dedicated SMPs. Specifically, we assume the absence of workload sharing, i.e., only one application runs on a node, and we enforce a one-to-one mapping between threads and processors. These assumptions are common for high-performance scientific computing [30, 31].

We make the following contributions in the paper. We have designed and implemented ccSIM, a cache-coherent simulator. We demonstrate good correlation between ccSIM results and hardware performance counters for a 4-way Power3 SMP node on a variety of OpenMP benchmarks. We obtain address traces per processor through dynamic binary rewriting. We demonstrate that ccSIM obtains detailed information indicating causes of invalidations and relates these events to their program location and data structures. This enables us to detect coherence bottlenecks and allows us to infer opportunities for optimizations.

The paper is structured as follows. We introduce the instrumentation framework METRIC that is utilized to obtain data traces. We then provide details on the design and implementation of ccSIM, the cache coherence simulator. Next, we describe the experimental methodology followed by simulation results as well as performance counter measurements. From these results, we derive opportunities for code transformations and assess their benefits. We conclude with related work and a summary of our contributions.

\section{Framework and Instrumentation}

Figure 1 depicts our simulation framework. Simulation of coherence traffic is based on two software components, a binary instrumentation tool to extract data traces from actual application executions and a cache simulator that consumes these traces to simulate coherence traffic. 


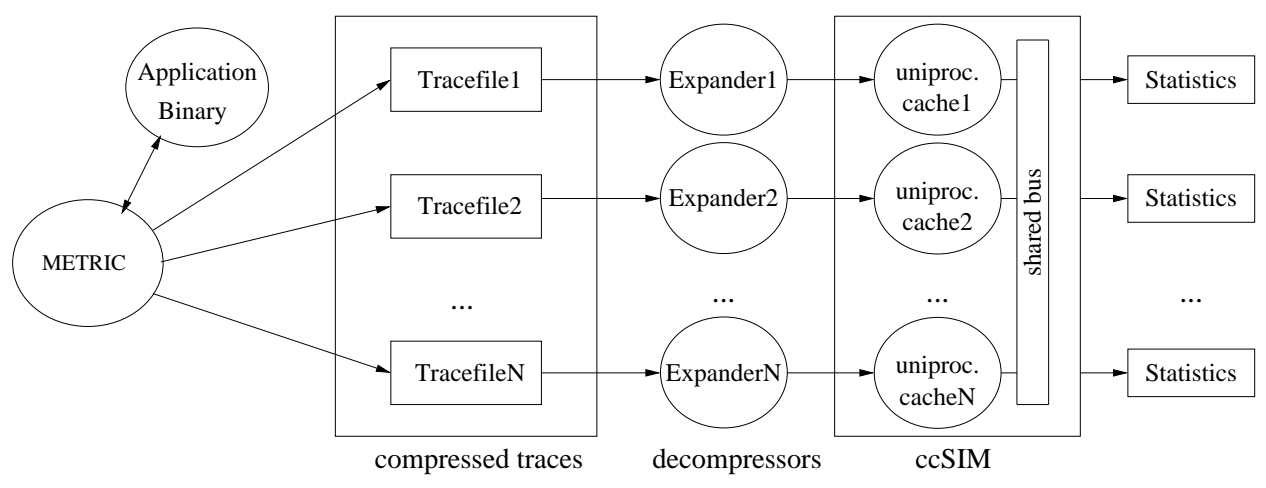

Figure 1: Overview of Framework

The instrumentation tool uses dynamic binary rewriting to instrument a running application. Memory accesses are instrumented to emit address reference information, including the relation of a reference to its source line in the program [21]. This information is compressed on-the-fly to conserve space before it is written to storage. Notice that the instrumentation does not affect the trace data, i.e., we collect the original trace addresses (even though performance is perturbed by the instrumentation). Later comparisons with performance counters are based on the uninstrumented application to ensure that the same work is being performed and instrumentation is excluded in the measurements.

The trace of each thread comprises the input to a uniprocessor cache hierarchy. Each cache hierarchy (corresponding to a separate processor) emits coherence messages on a shared bus according to the selected coherence protocol. Events, such as invalidation messages and cache misses, are being logged in the simulator and associated with source data structures as well as instruction locations in source files as they occur. This allows the generation of detailed statistics on a per-reference base with regard to invalidations, resulting misses and data subsequently evicted from cache.

Cache simulation for SMPs is based on address traces collected by METRIC, our framework for dynamic binary instrumentation of memory references [21]. METRIC inserts probes through a control program into an executing application to generate highly compressed address traces.

The process of dynamic binary rewriting operates as follows. A control program instruments the target OpenMP application executable using our customized extensions of the DynInst binary rewriting API [5]. These customizations, part of the METRIC framework, have been further extended to capture traces of OpenMP threads for this work. For each OpenMP thread, the memory access points (i.e., the loads and stores) are instrumented to capture the application access trace. To reduce the overhead on target execution, METRIC can trade off simulation accuracy for tracing speed by instrumenting only floating point or integer accesses. It also allows certain accesses such as local stack accesses to be ignored, since they often do not perceptibly affect the overall access metrics of the target program.

Once the instrumentation is complete, the target is allowed to continue. The trace logging of the access stream of each OpenMP thread proceeds in parallel without interaction with other OpenMP threads, thus increasing the tracing speed. For each thread, the instrumentation code calls handler functions in a shared library. The handler functions compress the generated trace online and write the compressed trace to stable storage.

For each thread, the accesses generated are compressed using the compression primitives described in our previous work [21]. Each compression primitive has a unique sequence_id field, which is globally unique for that thread, and anchors the compression primitive in the overall access stream for that thread. OpenMP supports SMP parallelism via compiler directives (\#pragma omp or ! \$OMP). We instrument the compiler-generated functions implementing these directives.

\section{3. ccSIM: A Multi-Processor Cache Simulator}

The compressed access trace generated from the instrumented OpenMP application is used for incremental multiprocessor memory hierarchy simulation. We have designed and implemented a memory access simulator for cache coherent shared-memory multiprocessor systems. The uniprocessor components were derived from MHSim [24].

The ccSIM tool feeds each trace file into a driver object, each of which corresponds to a separate processor (see Figure 1). Such a trace contains entries corresponding to the sequence of events during its execution, e.g, data references as well as OpenMP directives. Each driver feeds an instance of a uniprocessor cache hierarchy. During the simulation, fine-grained statistics on cache metrics and coherence traffic are maintained on a per-reference and per-data structure basis. These statistics provide important feedback about the application behavior and performance. The causes of bottlenecks on the SMP architecture of interest can be determined from the information obtained.

\subsection{Interleaving of Reference Streams}

During application execution, the actual interleaving of data references is non-deterministic between synchronization points. However, at synchronization points, such as barriers, threads are guaranteed to eventually reach a certain point of execution within the program. We study the effect of different reference interleavings by supporting two simulation modes. Recall that each OpenMP thread is assumed to be executing on a separate processor. Hence, every driver object maps to a unique processor on an SMP node.

ccSIM implements the semantics of OpenMP constructs that affect the execution order of threads at synchronization points, i.e., barriers, critical sections, atomic sections and accesses protected by explicit mutex locks (omp_get_lock, omp_set_lock). Entry and exit events for these constructs are recorded in the trace for each thread.

We refer to the program code between two synchronization points in an SPMD model as a region. At the start of a region, the simulator can operate in one of two modes.

Interleaved Mode: The simulator processes one data reference from each trace (corresponding to a thread or processor) before processing the second reference for each trace etc. Effectively, the simulator enforces a fine-grained interleaving in a round-robin fashion on a per-reference base in this mode. 
Piped Mode: The simulator processes all data references from one trace up to the next synchronization point before processing data references from the second trace etc., effectively enforcing a coarse-grained interleaving at the level of regions.

A comparison of results from the interleaved and piped modes reflects the extent to which program latency is affected by the nondeterministic order of execution of OpenMP threads and may provide extremes (bounds) on metrics for coherence traffic.

Example: Figure 2 shows the trace events and simulator actions for a simple OpenMP program with two active OpenMP threads. A and B are shared arrays of size N, and $i$ is a local variable. Static loop scheduling is assumed for the OpenMP for loop. The entry into the parallel OpenMP region is logged as a trace event and causes the simulator to activate two driver objects. Accesses generated by each OpenMP thread to the A and B arrays are logged separately. The drivers may simulate these accesses in parallel, as shown for the interleaved mode. When an OpenMP thread exits from the implicit barrier at the end of the for loop, a barrier exit event is logged for that thread. Detection of a barrier event causes drivers to synchronize. Another synchronization takes place when the parallel end event is processed. After an OpenMP parallel region, a serial phase starts, and only one driver (corresponding to the master thread) will remain active. All others remain unused till the start of the next parallel phase.

For each thread, the address of the memory access is mapped to the unique machine instruction location that generated that access. The access address is also mapped to the language-level data structure to which it belongs. These mappings allow us to tag cache access and coherence statistics with higher level abstractions, such as line numbers and source code data structure identifiers.

\subsection{Studying Invalidations and Misses}

A key metric for the identification of memory performance bottlenecks in a multiprocessor system is the number of invalidations to lines in the lowermost level of cache of each processor. This is a major source of coherence traffic, potentially causing the shared bus to be a bottleneck in a symmetric multiprocessor architecture. More significantly, these invalidations could lead to coherence misses, thus increasing memory latency. It is these invalidation misses that we focus on in our experiments since an increasing number of invalidations leading to coherence misses can greatly hamper performance.

Since the main motivation in reducing the invalidate traffic is to decrease the number of coherence misses, it is imperative to distinguish between coherence misses and uniprocessor misses in a processor. Invalidations to cache lines can further be classified as true-sharing invalidations and false-sharing invalidations in each level of cache. True-sharing invalidations arise from accesses to the same shared memory location by more than one processor, with at least one access being a write access. False-sharing invalidations are caused due to accesses to different memory locations that map to the same cache line on more than one processor. ccSIM maintains state between accesses to a cache line to detect and distinguish true/false sharing. This level of classification gives a better view of the causes of the invalidations, which helps in determining the applicability of various techniques for optimizations.

With respect to OpenMP parallel programs, another level of classification can be introduced, which is instrumental in determining the feasibility of using certain optimization techniques to reduce the coherence traffic. This involves determining whether the invalidations to cache lines occur due to references across synchronization points or between synchronization points in a parallel program (Figure 3). References across processors leading to true sharing invalidations within a region can be distinguished as follows:

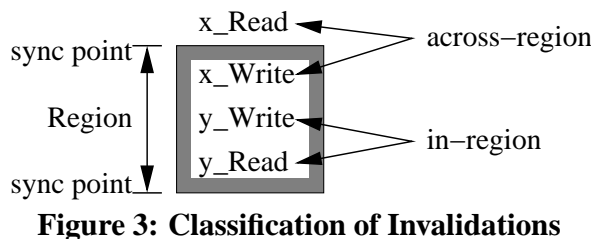

- References not protected by locks: These typically occur in the single-writer, single/multiple-reader scenario where one processor writes to a common location and one or more processors read from it.

- References protected by locks: These typically occur in the multiple-writer, single/multiple-reader scenario where multiple processors write and read from a common location.

In addition to these metrics, the simulator also generates perprocessor statistics for hits, misses, temporal and spatial locality, and eviction-related information.

For each of the above-mentioned metrics, aggregate numbers for the application help in an overall analysis of the observed performance. A further breakdown of these statistics for each reference or for each data structure in the program provides deeper insight into the behavior of the application. Statistics are computed for each of the globally shared data structures in order to provide information at a greater level of detail and to determine the exact causes of inefficiencies in the memory hierarchy. This enables us to pinpoint the data structures contributing to latency caused by coherence misses. A detailed analysis of the compiled metrics helps in determining the particular choice of optimization techniques for a benchmark.

\section{Experiments}

First, we present the OpenMP benchmarks used for experiments with ccSIM. Next, we discuss ccSIM comparison with hardware performance counters. We then use ccSIM to characterize the shared memory usage of representative OpenMP benchmarks and show how ccSIM statistics are useful in detecting and isolating coherence bottlenecks.

Benchmarks: We selected 7 OpenMP benchmarks for our experiments. Out of these - IS, MG, CG, FT, SP and BT are from the NAS OpenMP benchmark suite [15]. NBF is a part of GROMOS[13]. A brief description of each benchmark is given below.

1. IS: A large integer sort used in "particle method" codes.

2. MG: A V-cycle MultiGrid method to compute the solution of the 3-D scalar Poisson equation.

3. CG: A Conjugate Gradient method to compute an approximation to the smallest eigenvalue of a large, sparse, unstructured matrix.

4. FT: An implementation of a 3-D Fast Fourier Transform (FFT)-based spectral method.

5. SP: A simulated CFD application with scalar pentagonal bands of linear equations that are solved sequentially along each dimension.

6. BT: A simulated CFD application with block tridiagonal systems of $5 \times 5$ blocks solved sequentially along each dimension.

7. NBF (Non-Bonded Force Kernel): A molecular dynamics simulation computing non-bonded forces due to molecular interactions.

Comparison with Hardware Counters: Next, we validate ccSIM against measurements from hardware performance counters. From a developer's perspective, the number of coherence misses is the most important facet of the shared memory access pattern of an application. However, there are no hardware counters capable of measuring coherence misses on our target platform. Instead, we 


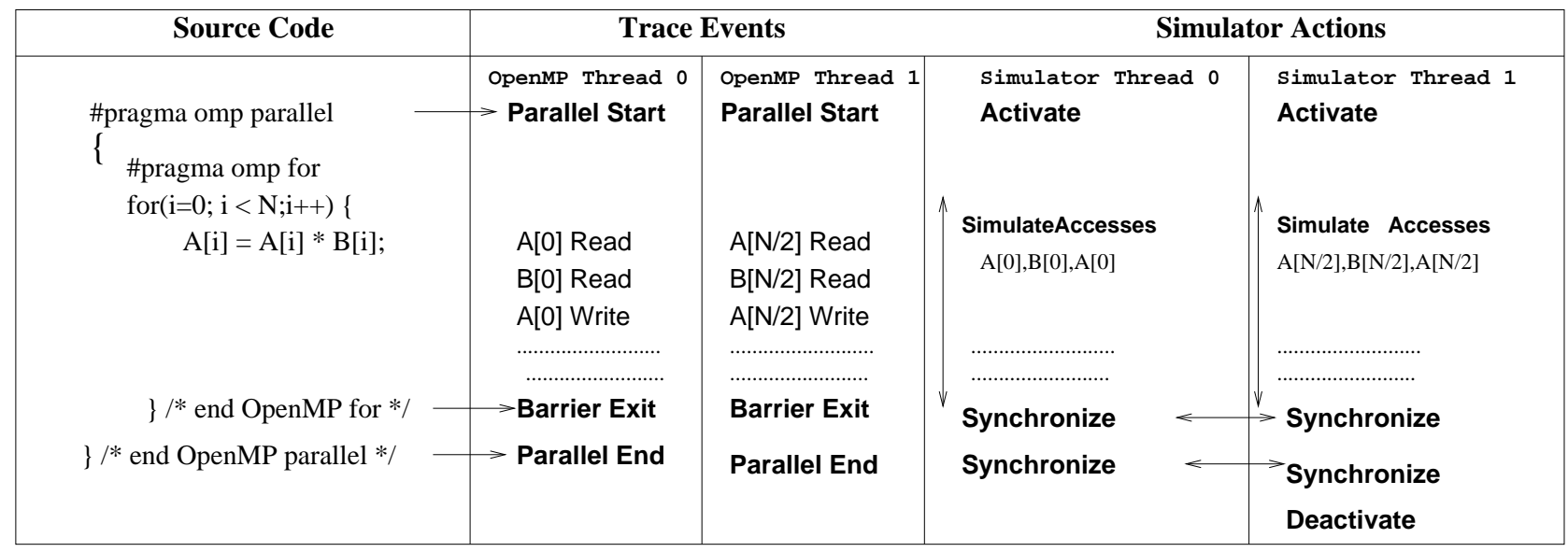

Figure 2: Illustration: Trace Events and Simulator Actions

compare the number of invalidations for ccSIM against the actual number of invalidations measured by the hardware counters. The total number of invalidations is an upper bound on the number of coherence misses for the application. Reducing invalidations will also lower the number of coherence misses, thereby improving application performance.

Hardware Environment: The hardware counter measurements were carried out on a single 8-way node of a 4-way Power3 SMP node. The hardware counters were accessed through the proprietary Hardware Performance Monitor (HPM) API. The system has a $64 \mathrm{~KB} 128$-way associative $\mathrm{L} 1$ cache with round-robin replacement and an $8 \mathrm{MB} 4$-way associative L2 cache. All experiments were carried out with 4 active OpenMP threads bound to distinct processors. The IBM OpenMP compilers, xlc_r and xlf_r, were used to compile the benchmarks at the default optimization level $\mathrm{O} 2$ with following flags settings: -qarch=auto, -qsmp=omp, -qnosave.

HPM measurements: The Power3 hardware implements the MESI coherence protocol within an SMP node. The PM_SNOOP_L2_E_OR_S_TO_I and PM_SNOOP_M_TO_I HPM events were used to measure the number of L2 cache invalidations with $\mathrm{E} \rightarrow \mathrm{I}, \mathrm{S} \rightarrow \mathrm{I}$ and $\mathrm{M} \rightarrow \mathrm{I}$ transitions, respectively. The OpenMP runtime system also contributes to the number of invalidations measured. Since we are interested only in the invalidations of the application, we need to remove these invalidations from the measured numbers.

To assess the side-effect of the OpenMP runtime system on invalidations, we measured invalidations for OpenMP runtime constructs with empty bodies in a set of microbenchmarks. For example, the overhead in terms of invalidations for a barrier construct was determined. The microbenchmarks were subsequently used to adjust raw HPM data obtained from application runs by removing the extrapolated effect of OpenMP runtime invalidations for $n$ iterations. For example, we removed the effect of $n=100$ times the overhead for a single barrier if the benchmark contained 100 barriers. We refer to these measurements as the raw HPM metrics and the OpenMP-adjusted HPM metrics.

Table 1 shows the raw and OpenMP-adjusted HPM measured invalidations for the $\mathrm{L} 2$ cache. The invalidations were measured for each processor separately using the HPM events discussed above and summed up to get the total invalidations shown in the table. Each HPM measurement is the mean of 5 samples.

Comparison with ccSIM: ccSIM was configured with the MESI coherence protocol and with the cache parameters of the hardware platform (4-way Power3 SMP node). Both L1 and L2 caches were
Table 1: Total L2 invalidations with HPM

\begin{tabular}{|r|r|r|}
\hline Benchmark & HPM(raw) & HPM(OpenMP-adjusted) \\
\hline IS & 165246 & 162964 \\
\hline MG & 24631 & 13629 \\
\hline CG & 134964 & 100488 \\
\hline FT & 326595 & 325257 \\
\hline SP & 282269 & 258923 \\
\hline BT & 185317 & 157384 \\
\hline NBF & 474121 & 135926 \\
\hline
\end{tabular}

simulated. Table 2 compares total L2 invalidations for HPM and the two ccSIM modes - piped and interleaved.

The results indicate a good correlation between ccSIM and HPM for most benchmarks. The absolute error between ccSIM and HPM is less than $17 \%$ for all benchmarks and less than $7 \%$ for most. Moreover, for the NAS benchmarks, both interleaved and piped modes result in closely matching numbers of invalidations. This indicates that for these benchmarks, fine-grained round-robin simulation is not necessary to achieve a high level of simulation accuracy. NBF stands out as an anomalous case with significant difference between the interleaved and piped modes of simulation. ccSIM allows us to categorize invalidations into true and false sharing invalidations as well as to distinguish between across-region and in-region invalidations, as explained in Section 3.2. The cause of the discrepancy becomes apparent when we examine the in-region true-sharing critical invalidations shown in Figure 4. Metrics are plotted in a log scale. The number of true-share invalidations occurring within a region is much higher (at least an order of magnitude) in the interleaved simulation mode. The interleaved simulation mode involves fine-grained round-robin simulation, which leads to "ping-ponging" of shared data across processors. Pingponging does not take place with the piped mode of simulation, leading to a very small number of invalidations to be recorded. A look at the per-reference ccSIM statistics indeed shows that the most significant invalidation source is a data access point inside an OpenMP critical construct. This demonstrates the necessity of interleaved simulations for codes containing critical sections to closely resemble the interleaving of references during actual execution. Next, let us consider the detailed results for two benchmarks and general trends for the benchmark suite.

\section{Characterization of Benchmarks}

Our simulation results are not only accurate with respect to actual executions, we also obtain detailed classifications indicating the cause of invalidations as well as the corresponding location 


\begin{tabular}{|c|c|c|c|c|c|c|c|}
\hline Benchmark & IS & MG & CG & FT & $\overline{\mathrm{BT}}$ & SP & $\mathrm{NBF}$ \\
\hline HPM & 162964 & 13629 & 100487 & 325257 & 157384 & 258922 & 135926 \\
\hline ccSIM-Intl & 163073 & 13174 & 117117 & 302630 & 157503 & 268334 & 137498 \\
\hline ccSIM-Pipe & 159913 & 12355 & 116318 & 302607 & 157480 & 268334 & 14629 \\
\hline$\%$ Error(Interleaved vs. HPM) & -0.06 & 3.3 & -16.5 & 6.9 & -0.07 & -3.6 & -1.15 \\
\hline
\end{tabular}

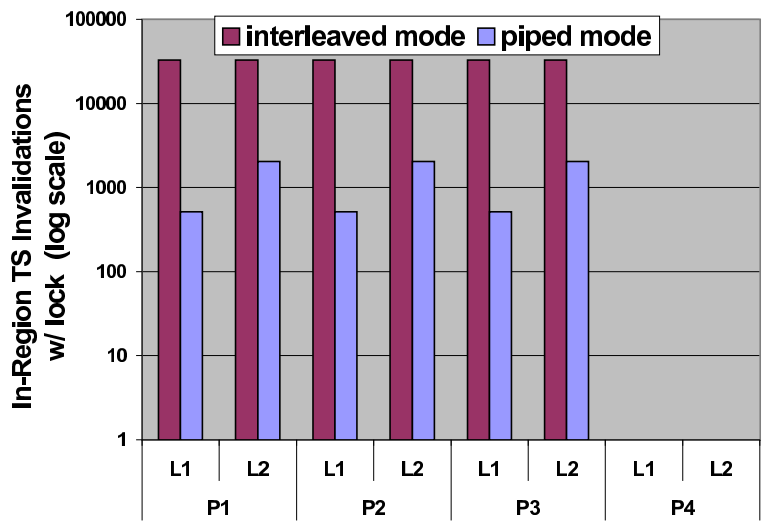

Figure 4: NBF: Interleaved and Piped

in the program. Due to space limitations, we discuss only MG and NBF. The former is representative in large for any of the first six benchmarks while the latter has a unique coherence footprint, which indicates room for optimizations subsequently discussed.

\subsection{MG and General Trends}

Figures 5(a) to 5(e) represent the behavior of this benchmark observed by ccSIM. Figure 5(a) shows that coherence misses are rare in L1 while they constitute 55-63\% of total misses in the second level of cache. The size of the $\mathrm{L} 1$ cache causes uniprocessor misses to completely dominate the misses occurring in this level. The total misses and coherence misses are almost uniform across processors, which is common for SPMD programming styles. Small variations are typically due to imbalanced sharing of data across sharing boundaries, such as in stencil problems and grid-based calculations. Inner processors have more neighbors resulting in larger number of invalidations. Hence, the number of invalidations depicted in Figure 5(b) is higher in processors two and three since this metric amplifies these variations. Detailed access simulation by ccSIM also allows us to distinguish the cause of invalidations as true-sharing and false-sharing invalidations, shown in Figure 5(b). Interestingly, a significant fraction of the total invalidations are false-sharing invalidations. Within these classes, we can further determine whether invalidations resulted from two references crossing a synchronization point (across multiple regions) or not (within a synchronization region), depicted in Figure 5(c). True-sharing invalidations mainly arise from references occurring across regions. A majority of the false-sharing invalidations also occur across region boundaries. We can further classify in-region invalidations into two classes: Those due to references within a critical section (while holding a lock) and those outside of critical sections (without holding a lock). Figure 5(d) indicates that only an insignificant number of in-region true-sharing invalidations occur, and these invalidations are due to accesses without locks. (While this metric is not indicative for MG, it is significant for NBF discussed in the following and depicted for symmetry.) Finally, not all invalidations may lead to subsequent misses, but ccSIM allows us to determine if an invalidate is followed by a miss, as depicted in Figure 5(e). The percentage of invalidations leading to misses is significant (around 50-70\%) in the L1 cache and very high (approximately 95\%) in the L2 cache.

For the other benchmarks, with the exception of NBF, we observe similar trends in the ratio between total misses and coherence misses and the ratio between L1 and L2 misses. We find that a significant fraction of invalidations are false-sharing invalidations, a majority of which cross region boundaries. Most true-sharing invalidations also cross region boundaries. Finally, a large portion of invalidations, particularly in L2, will subsequently result in a coherence miss. Hence, the results discussed for MG are representative for the other benchmarks as well - with the exception of NBF discussed in the following.

\subsection{NBF: Non-Bonded Force Kernel}

Figures 6(a) to 6(e) represent the results obtained from ccSIM by simulated execution of this benchmark. NBF contains a critical section with updates of shared data inside a parallel region. From figure 6(a), we observe that a significant percentage of misses in L1 and L2 caches are coherence misses. Almost all invalidations are true-sharing invalidations in both the L1 and L2 caches, except for processor 4 (Figure 6(b)) where false-sharing invalidations dominate. This is an artifact of the order of access scheduling in the fine-grained round-robin ccSIM simulation. As we shall see in the next section, almost all invalidations occur in a loop updating a global shared array, executed by every processor. Since the processors are ordered by their logical ids, the array element written to by processor- 1 is different from the element accessed by processor- 4 in the last iteration, but they are adjacent in the L1/L2 cache line. This causes the resulting invalidation (since processor- 1 is writing) to be classified as a false-sharing invalidation for processor-4's caches. In an execution with non-deterministic ordering, these false sharing invalidations will still occur but will be distributed evenly over the caches of all processors. Figure 6(c) shows that almost all of the true-sharing invalidations take place within regions. Locks protect the references that cause true-sharing invalidations within regions (see Figure 6(d)). This observation is central to the opportunities for optimizations discussed in the next section. An important characteristic is the percentage of invalidations resulting in coherence misses, shown in Figure 6(e). We see that more than 95\% of the invalidations subsequently caused a coherence miss. The coherence results obtained for NBF indicate opportunities for optimizations with respect to in-region true-sharing with locks (critical sections), but only more detailed simulation can provide conclusive information to determine beneficial transformations, as discussed in the following.

\section{Opportunities for Transformations}

In this section, we demonstrate how ccSIM can be used to detect and isolate coherence traffic bottlenecks, and we derive opportunities for transformations leading to reduced coherence traffic and, thereby, potential performance gains.

\subsection{NBF: Non-Bonded Force Kernel}

We first discuss the NBF kernel described in the previous section. A full access trace was obtained for the OpenMP NBF kernel. The OpenMP environment was set to four threads and static scheduling (OMP_NUM_THREADS $=4$, OMP_SCHEDULE_STATIC).

Analysis: Consider the results for NBF again. Figure 6(a) shows the breakdown of misses for L1 and L2 caches for each processor obtained by ccSIM.

We observe that almost all L2 misses and a significant number of 


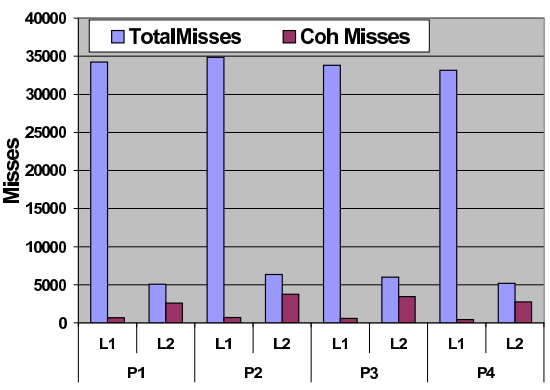

(a) MG: Misses

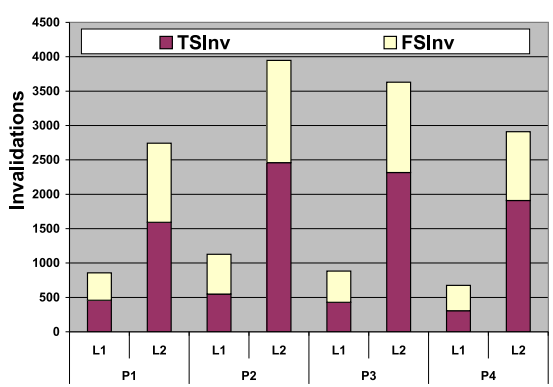

(b) MG: Invalidations

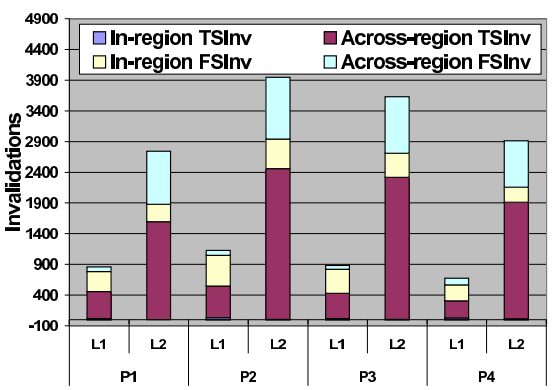

(c) MG: Region-wise Classification of Invalidations

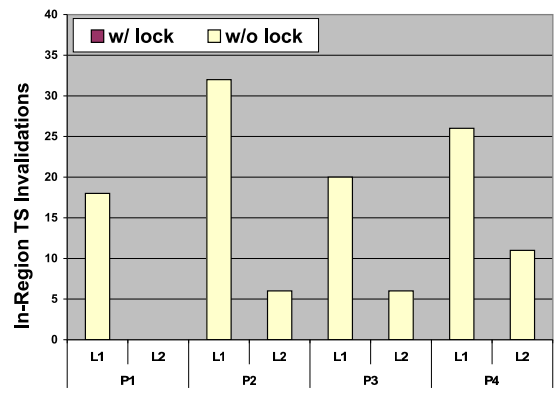

(d) MG: In-Region True-Sharing Invalidations

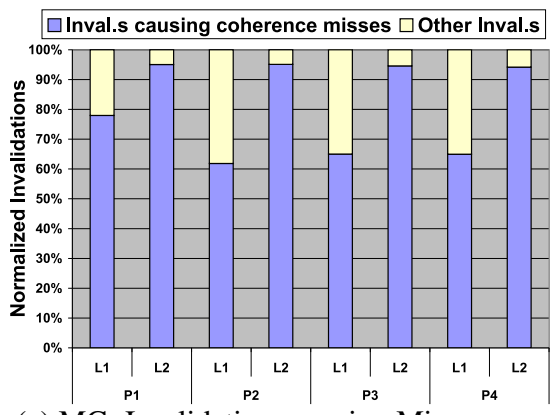

(e) MG: Invalidations causing Misses

Figure 5: Results for MG

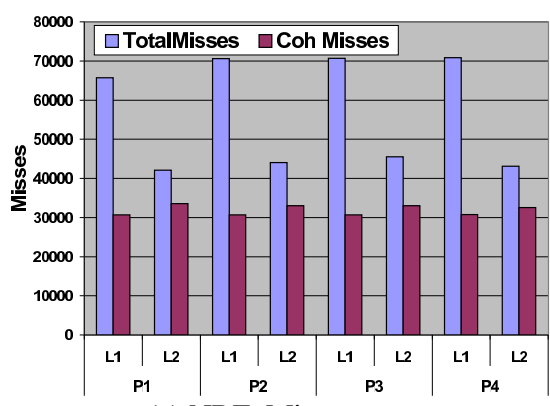

(a) NBF: Misses

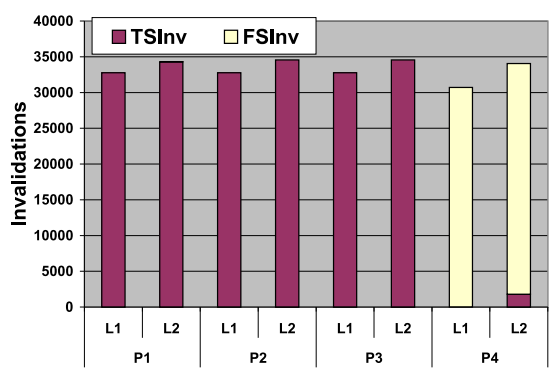

(b) NBF: Invalidations

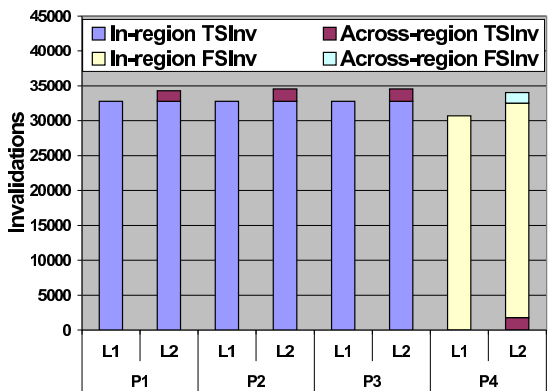

(c) NBF: Region-wise Classification of Invalidations

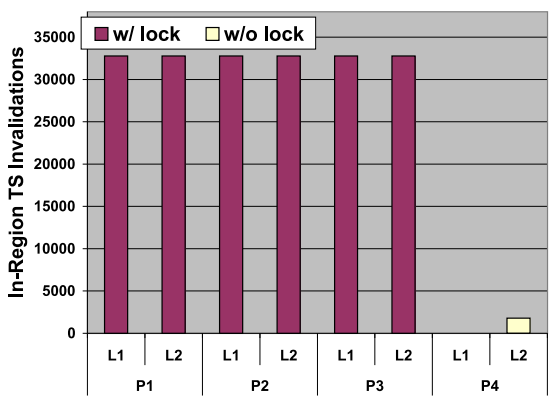

(d) NBF: In-Region True-Sharing Invalidations

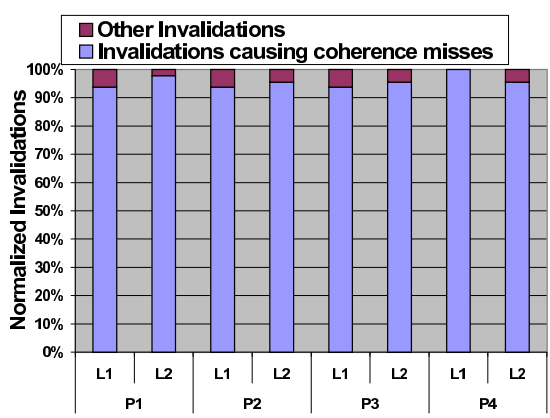

(e) NBF: Invalidations causing Misses

Figure 6: Results for NBF 
Table 3: NBF: Comparison of per-reference statistics for each optimization strategy

\begin{tabular}{|c|c|c|c|c|c|c|c|c|c|c|}
\hline \multirow{3}{*}{$\begin{array}{l}\text { Line } \\
\text { No. }\end{array}$} & \multirow{3}{*}{ Ref } & \multirow{3}{*}{$\begin{array}{l}\text { Optimization } \\
\text { Strategy }\end{array}$} & \multirow{3}{*}{ Misses } & \multirow{3}{*}{$\begin{array}{l}\text { Miss } \\
\text { Ratio }\end{array}$} & \multirow{3}{*}{$\begin{array}{c}\% \text { Coherence } \\
\text { Misses }\end{array}$} & \multicolumn{5}{|c|}{ Invalidations } \\
\hline & & & & & & \multirow[b]{2}{*}{ Total } & \multicolumn{2}{|c|}{ True } & \multicolumn{2}{|c|}{ False } \\
\hline & & & & & & & $\begin{array}{c}\text { In } \\
\text { Region }\end{array}$ & $\begin{array}{l}\text { Across } \\
\text { Region }\end{array}$ & $\begin{array}{c}\text { In } \\
\text { Region }\end{array}$ & $\begin{array}{l}\text { Across } \\
\text { Region }\end{array}$ \\
\hline \multirow[t]{3}{*}{$\overline{141}$} & \multirow[t]{3}{*}{$\overline{\text { f_Read }}$} & Original & $\overline{32500}$ & 0.99 & $\overline{96.87 \%}$ & $\overline{\mathbf{3 2 7 6 8}}$ & $\overline{32768}$ & $\overline{0}$ & $\overline{0}$ & $\overline{00}$ \\
\hline & & Serialized & 2050 & 1.0 & $50.30 \%$ & 2048 & 2048 & 0 & 0 & 0 \\
\hline & & Round-robin & 1790 & 0.87 & $42.84 \%$ & 2048 & 2048 & 0 & 0 & 0 \\
\hline \multirow[t]{3}{*}{227} & \multirow[t]{3}{*}{ x_Read } & Original & 1540 & 0.997 & $99.74 \%$ & 768 & 1 & 765 & 0 & 2 \\
\hline & & Serialized & 1540 & 0.997 & $99.74 \%$ & 768 & 1 & 765 & 0 & 2 \\
\hline & & Round-robin & 1540 & 0.997 & $99.74 \%$ & 768 & 0 & 766 & 0 & 2 \\
\hline \multirow[t]{3}{*}{217} & \multirow[t]{3}{*}{ f_Read } & Original & 512 & 1.0 & $100 \%$ & 256 & 256 & 0 & 0 & 0 \\
\hline & & Serialized & 512 & 1.0 & $100 \%$ & 256 & 256 & 0 & 0 & 0 \\
\hline & & Round-robin & 512 & 1.0 & $100 \%$ & 256 & 0 & 255 & 0 & 1 \\
\hline
\end{tabular}

L1 misses are coherence misses. A coherence miss is caused when a processor accesses a cache line that was invalidated due to a write from another processor. However, a large number of invalidations does not necessarily imply a large number of coherence misses, since the invalidated cache lines may not be referenced by the processor again before being flushed out of the cache. The breakup of invalidations in Figure 6(e) shows that a significant number of invalidations resulted in coherence misses, especially in L2 cache. This indicates that minimizing the total number of invalidations will reduce the magnitude of coherence misses correspondingly.

We have detected a coherence bottleneck. We can use the perreference coherence and cache statistics generated by ccSIM to determine the cause of the bottleneck. Table 3 shows the perreference statistics on processor one for the top three references of the original code and two optimization strategies (serialized and round-robin) discussed next. Only L2 cache statistics are shown.

We observe that access metrics across all processors are uniform. The f_Read reference on line 141 of the source code has an exceptionally high miss rate in all processors. Moreover, more than $96 \%$ of the misses for this reference are coherence misses. The invalidation data shows that the large number of in-region invalidations are the primary cause for these misses. The relation of this reference to the source code indicates that line 141 is of interest:

\#pragma omp parallel

...

for $(i=0 ; i<$ natoms; $i++)\{$

\#pragma omp critical

141: $f[i]=f[i]+f l o c a l[i]$;

\}

The for loop updates the global shared array $f$ with values from the local private copy flocal for each OpenMP thread. The large number of invalidations attributed to the $f \_R e a d$ reference is due to the ping-ponging of the shared $f$ array between processors as all of them try to update the global $f$ array simultaneously.

Optimizing Transformations: Using ccSIM's per-reference statistics, we isolated the coherence bottleneck to the updates of the shared global array $f$. We shall discuss two ways of reducing the number of coherence misses. One method eliminates the ping-ponging of the $f$ array by serializing the updates to the array $f$ since they require mutually exclusive writes. This is achieved by moving the critical section to encompass the entire for loop instead of the single update. The modified code is shown below.

\#pragma omp parallel

...

\#pragma omp critical

for $(i=0 ; i<$ natoms; $i++) \quad\{$

$f[i]=f[i]+f l o c a l[i] ;$ \}

Moving the critical statement outside the loop also reduces the number of times that the mutual exclusion region must be entered and exited, decreasing the execution overhead.
Although reducing the number of coherence misses, this method does not exploit the potential for parallel updates to separate parts of the $f$ array by different threads. Hence, we consider an alternate transformation. We can exploit parallelism by partitioning the array $f$ into a number of segments. Each thread updates a distinct segment until all segments are updated. We call this scheme the round-robin update scheme. The modified code is shown below as pseudo-code.

$i=0$;

for each thread \{

1. segment_number $=i+$ thread_id;

2. update segment

3. synchronize w/ other threads (barrier)

4. $i=(i+1)$ MOD max segments

\}//run till all segments are updated

Results: Table 3 compares the L2 coherence misses and invalidations for the two optimization strategies. Statistics are depicted only for processor- 1 and are similar for the other processors. We observe that both strategies lead to a significant decrease in the volume of coherence misses for the $f_{-}$Read reference. Table 5 shows the wall-clock execution time for (a) the routine that updates the shared array $f,(b)$ the remainder and (c) the entire program. We observe that the transformations cause a significant improvement in wall-clock execution time. Table 6 shows the total L2 invalidations for each approach measured with HPM. It confirms that the actual number of invalidations occurring in the hardware indeed decreases by an order of magnitude for the serialized and round-robin schemes over the original program.

Table 5: NBF: Wall clock Times (Seconds)

\begin{tabular}{|l|l|l|l|}
\hline $\begin{array}{l}\text { Code } \\
\text { Segment }\end{array}$ & Original & Serialized & Round-robin \\
\hline f-Update & 4.981 & $0.003(\mathbf{9 9 . 9 \%})$ & $0.003(\mathbf{9 9 . 9 \% )}$ \\
Other & 2.141 & $2.076(3 \%)$ & $2.190(-2.28 \%)$ \\
Overall & 7.122 & $2.079(\mathbf{7 0 . 8 \%})$ & $2.193(\mathbf{6 9 . 2 \%})$ \\
\hline
\end{tabular}

Table 6: NBF: L2 Invalidations (HPM raw)

\begin{tabular}{|l|r|r|r|}
\hline Code & Original & Serialized & Round-robin \\
Segment & & & \\
\hline f-Update & 503654 & 921 & 6209 \\
Other & 37987 & 32916 & 38863 \\
Overall & 541641 & 33837 & 45072 \\
\hline
\end{tabular}

\subsection{IRS: Implicit Radiation Solver}

IRS-1.4 is part of the ASCI Purple codes [1]. IRS can use MPI, OpenMP or a mixture of both for parallelization. We use the pure OpenMP version of IRS for our study. Existing OpenMP parallelization uses "omp parallel do" constructs for loop level parallelization. For the analysis below, we ran IRS for 10 calls to the top-level xirs function, with a limited data set (NDOMS $=10$, 
Table 4: IRS: Per-Reference Statistics

\begin{tabular}{|c|c|c|c|c|c|c|c|c|c|c|}
\hline \multirow{3}{*}{ Proc } & \multirow{3}{*}{ No. } & \multirow{3}{*}{ Reference } & \multirow{3}{*}{ Group } & \multirow{3}{*}{$\begin{array}{r}\text { Coherence } \\
\text { Misses }\end{array}$} & \multicolumn{4}{|c|}{ Invalidations } & \multirow{3}{*}{$\begin{array}{l}\text { Optimization } \\
\text { Strategy }\end{array}$} & \multirow{3}{*}{$\begin{array}{r}\text { Optimized } \\
\text { Coherence } \\
\text { Misses }\end{array}$} \\
\hline & & & & & \multicolumn{2}{|c|}{ True } & \multicolumn{2}{|c|}{ False } & & \\
\hline & & & & & In & Across & In & Across & & \\
\hline \multirow[t]{7}{*}{1} & 1 & v1[]_Read & \multirow{4}{*}{1} & 8627 & 4 & 7517 & 31 & 1342 & \multirow{4}{*}{$\begin{array}{l}\text { Code Transforms } \\
\text { for data } \\
\text { segregation }\end{array}$} & 1980 \\
\hline & 2 & v2[]_Read & & 8568 & 310 & 5093 & 78 & 3085 & & 1971 \\
\hline & $3-16$ & matrix.dbl[]_Write & & 2547 & 25 & 2325 & 0 & 455 & & 719 \\
\hline & 17 & $\mathrm{x}[] \_$Read & & 1803 & 0 & 1402 & 391 & 2 & & 968 \\
\hline & 18 & timersflgg Read & \multirow[t]{2}{*}{2} & 3182 & 1 & 0 & 3122 & 70 & \multirow[t]{2}{*}{ Padding } & 0 \\
\hline & 19 & thread_fbp[] Read & & 1789 & 0 & 2 & 1789 & 0 & & 0 \\
\hline & 20 & clock_last_Read & 3 & 2165 & 2166 & 0 & 0 & 0 & Remove Sharing & 0 \\
\hline \multirow[t]{5}{*}{2} & 1 & clock_last_Read & 3 & 5997 & 5644 & 353 & 0 & 0 & & $\mathbf{0}$ \\
\hline & $2-3$ & timersflgg Read & \multirow{4}{*}{2} & 2907 & 18 & 0 & 2908 & 0 & \multirow{4}{*}{ Padding } & $\mathbf{0}$ \\
\hline & $4-6$ & thread_fbp[] Read & & 2734 & 0 & 0 & 2407 & 327 & & $\mathbf{0}$ \\
\hline & 7 & thread_wall_secs[]_Read & & 1022 & 0 & 0 & 652 & 371 & & $\mathbf{0}$ \\
\hline & 8 & thread_cpu_secs[]_Read & & 811 & 0 & 71 & 742 & 0 & & $\mathbf{0}$ \\
\hline
\end{tabular}

Table 7: SMG: Per-Reference Statistics (Processor-1)

\begin{tabular}{|c|c|c|c|c|c|c|c|c|c|}
\hline \multirow{3}{*}{ No. } & \multirow{3}{*}{ Reference } & \multirow{3}{*}{ Group } & \multirow{3}{*}{$\begin{array}{r}\text { Coherence } \\
\text { Misses }\end{array}$} & \multicolumn{4}{|c|}{ Invalidations } & \multirow{3}{*}{$\begin{array}{l}\text { Optimization } \\
\text { Strategy }\end{array}$} & \multirow{3}{*}{$\begin{array}{r}\text { Optimized } \\
\text { Coherence } \\
\text { Misses }\end{array}$} \\
\hline & & & & \multicolumn{2}{|c|}{ True } & \multicolumn{2}{|c|}{ False } & & \\
\hline & & & & In & Across & In & Across & & \\
\hline 1 & rp[]_Read & $\mathbf{1}$ & 170046 & 0 & 0 & $\overline{156585}$ & 13387 & Code Transforms & 256 \\
\hline 2 & rp[]_Read & & 83509 & 0 & 0 & 80145 & 3529 & for coarse-level & $\mathbf{0}$ \\
\hline 3 & rp[]_Write & & 43640 & 0 & 0 & 43305 & 3373 & interleaving & $\mathbf{0}$ \\
\hline 4 & xp[]_Write & & 23193 & 0 & 0 & 22309 & 1284 & & 2764 \\
\hline 5 & num_threads & 2 & 44362 & 44929 & 0 & 0 & 0 & Remove sharing & $\mathbf{0}$ \\
\hline
\end{tabular}

ZONES_PER_SIDE=NDOMS_PER_SIDE) with 4 OpenMP threads and static scheduling. This partial data trace is comparatively small, yet captures essential coherence traffic. Once our optimizations are complete, we compare the wall-clock time for the recommended full-sized data set for IRS ( $\mathrm{zrad} .008$. seq).

Analysis: Figure 7 shows that for all processors, coherence misses constitute almost the entire volume of L2 cache misses. Interestingly, the coherence miss magnitudes are asymmetric with processor-1 experiencing more than twice the number of coherence misses of any another processor. Table 4 shows the per reference coherence statistics for processors 1 and 2. Statistics for other processors were similar to those for processor-2. References have been collected into groups with distinct coherence characteristics (Groups 1, 2 and 3). Multiple references are shown with only a single representative reference. For example, there are a set of fourteen references to different arrays in the matrix structure, all of which show similar coherence characteristics; these are represented by a single representative reference matrix.dbl [ ] in the table. We observe that the set of references with significant coherence behavior are quite different for processor- 1 and processor- 2 . We shall now analyze references belonging to each group in detail.

Group 1: These references account for the largest fraction of coherence misses. True sharing across-region invalidations are dominant for this group. This indicates that the data elements accessed by these references move across the L 2 caches of multiple processors. Consider the first two references (v1 [ ] and v2 [ ]). These references occur in the icdot function, which is only called at three locations from the MatrixSolveCG function. All call sites are in serial code, i.e., they are executed only by the master thread. Between successive calls, the argument arrays are updated by other processors in parallel regions:

/* only master */

for $(\mathrm{i}=0 ; \mathrm{i}<\mathrm{nblk} ; \mathrm{i}++)$

dotprev $+=\operatorname{icdot}(r[\mathrm{i}], \mathrm{z}[\mathrm{i}], \ldots) ; /$ Reads $\mathrm{r}, \mathrm{z} * /$

I* parallel updates to $\mathrm{r}, \mathrm{z} * /$

\#pragma omp parallel for

for $(\mathrm{i}=0 ; \mathrm{i}<$ nblk; i++) \{ $\operatorname{setpz} 1(\mathrm{r}[\mathrm{i}], \ldots) ; / *$ Writes to $\mathrm{r} * /$
$\operatorname{setpz} 1(\mathrm{z}[\mathrm{i}], \ldots) ; / *$ Writes to $\mathrm{z} * /$

\}$\ldots$

/* only master */

for $(\mathrm{i}=0 ; \mathrm{i}<\mathrm{nblk}$; i++)

$\operatorname{dotrz}+=\operatorname{icdot}(\mathrm{r}[\mathrm{iblk}], \mathrm{z}[\mathrm{iblk}], \ldots) ; / * \operatorname{Reads} \mathrm{r}, \mathrm{z} * /$

Thus parts of arrays $r$ and $z$ move between processor- 1 and other processors. We can eliminate this unnecessary movement using code transformations for data segregation. In this case, we can parallelize the icdot calls using OpenMP. This allocates segments of $r$ and $z$ arrays to specific processors, which eliminates unnecessary data movement. More significantly, icdot calls now operate in parallel, which potentially has a much bigger impact on performance than the elimination of data movement alone.

Similar transformations are carried out for other references from Group-1, which we do not further discuss here.

Group 2: In-region false sharing invalidations constitute almost the entire volume of invalidations for these references. The number of coherence misses closely matches the number of invalidations received. All these references are related to timer routines used for performance benchmarking. Most of the coherence misses arise due to parallel updates to counter arrays indexed by thread id. Since array elements are contiguous, this leads to false-sharing, causing ping-ponging of cache lines across processors. We use intra datastructure padding to align individual array elements at cache line boundaries, which eliminates coherence misses.

Group 3: This group has a single reference exhibiting large volumes of true in-region invalidations. These invalidations occur inside a omp critical region updating a shared global clock variable. We eliminate this sharing by maintaining clock variables for each thread separately.

Results: The coherence misses for each reference after optimization are shown in the last column of Table 4 . We see that coherences misses for Groups 2 and 3 have been eliminated (by padding and sharing elimination, respectively) and have decreased significantly for Group 1. Figure 8 shows the wall-clock execution times for the different optimization strategies on the recommended OpenMP data set. The readings were obtained on a non-interactive node with 8 OpenMP threads. DSeg represents 
code transformations for data segregation (Group 1 references). DSeg+Crit additionally removes the shared global clock (Group 3 reference). DSeg+Crit+Padding represents the fully optimized benchmark. We observe that DSeg causes significant decrease in wall clock execution time (over 30\%), compared to the original program. The performance impact is due to a combination of reduction in coherence traffic and the OpenMP parallelization on several serial code sections required for it.

\subsection{SMG2000: Semi-coarsening Grid Solver}

SMG2000 is part of the ASCI Purple codes [1]. The SMG code utilizes the hypre library [11], which can select between OpenMP and MPI parallelization. We use the default settings of SMG2000 for our analysis $(10 \times 10 \times 10$ grid, $c x=c y=c x=1.0)$. We then compare the wall-clock execution time for the recommended full-sized workloads for different optimization strategies.

Analysis: For all processors, the L2 miss rate is quite high, ranging from $64 \%$ to $81 \%$. Figure 9 shows that almost all of the L2 misses are coherence misses. The per-reference statistics for the top 5 references from processor- 1 are shown in Table 7. The statistics for other processors were similar to those of processor-1. As with IRS, we classify references into groups based on coherence characteristics to facilitate analysis.

Group 1: References in this group are all array access references. All references experience a very large volume of in-region false-sharing invalidations. This indicates that multiple processors are updating different data elements on the same cache line, causing the cache line to ping-pong between L2 caches of different processors. The cause of the large volume of invalidations lies in the suboptimal implementation of loop-level parallelization by the hypre library function. The function chooses a loop to out of a triply loop nest. Each loop in the nest iterates over a single coordinate axis. The order of iteration is $\mathrm{x}, \mathrm{y}, \mathrm{z}$ from the inner to the outer loop. The function always chooses the largest dimension for parallelization, with the default being the innermost loop ( $\mathrm{x}$ dimension). This results in fine-grained interleaving of thread accesses to adjacent array elements, resulting in large amounts of coherence traffic. To correct this, we hoist the OpenMP parallelization to the outermost loop ( $\mathrm{z}$ dimension) ensuring that threads access data on different cache lines.

Group 2: This group has a single store reference that exhibits large volumes of true-sharing in-region invalidations. The data element referenced is a shared variable which is simultaneously updated by all threads with the number of runnable OpenMP threads, inside an omp parallel construct. We eliminate this sharing by replacing the omp parallel construct with separate calls to omp_get_max_threads () in each thread.

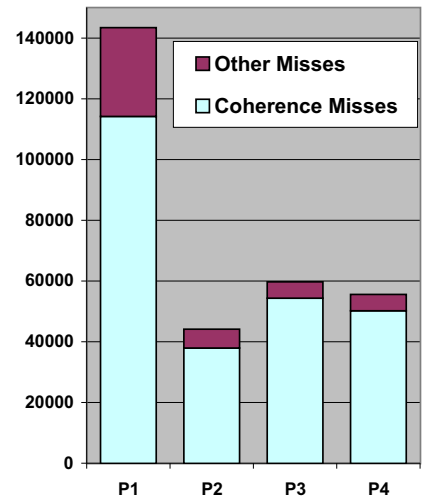

Figure 7: IRS: Breakdown of L2 misses

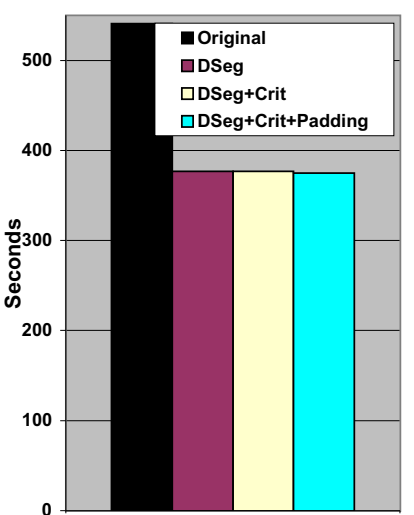

Figure 8: IRS: Time w/ 4 Optimizations

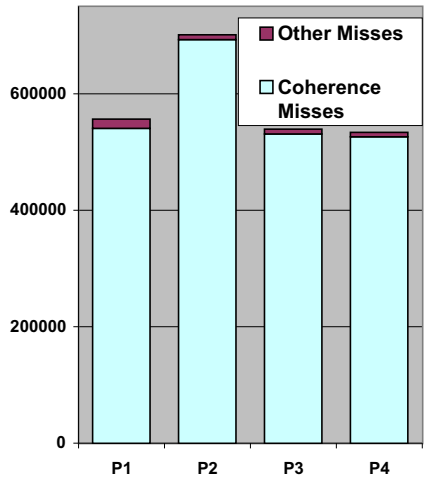

Figure 9: SMG: Breakdown of L2 misses

Results: The coherence misses after optimization are shown in the last column of Table 7. Our optimizations have eliminated almost all the coherence misses for these references. We compare the performance impact of our optimizations on wall clock execution time for the following workloads, as recommended by the SMG2000 benchmarking criteria:

1. $35 \times 35 \times 35$ grid, OpenMP threads $=1$

2. $35 \times 35 \times 70$ grid, OpenMP threads $=2$

3. $35 \times 70 \times 70$ grid, OpenMP threads $=4$

4. $70 \times 70 \times 70$ grid, OpenMP threads $=8$

All workloads have processor configuration $1 \times 1 \times 1 \quad\left(\begin{array}{lll}-\mathrm{P} & 1 & 1\end{array}\right.$ 1), $c x=0.1, c y=1.0, c z=10.0$. The workloads scale up the input grid size with increasing number of threads keeping the overall data processed per processor constant. Figure 10 compares the wall-clock times for the different workloads. Coarsening represents code transformations for coarse-level interleaving of accesses (Group 1). Coarsening+Sharing Removal additionally removes unnecessary shared data access (Group 2). We observe that both optimizations have significant impact on execution time, with a maximum improvement of $73 \%$ for the 4 th workload ( 8 OpenMP threads).

\section{Related Work}

There are several software-based and hardware-based approaches for memory performance characterization of shared memory multiprocessor systems. Gibson et al. provides a good overview of the trade-offs of each approach [12]. At one end of the spectrum are complete software machine simulators. RSim is a simulator for ILP multiprocessors with support for CC-NUMA architectures with a invalidation-based directory mapped coherence protocol [14]. SimOS is a complete machine simulator capable of booting commercial operating systems [27]. However, these frameworks simulate hardware and architecture state to a great detail,

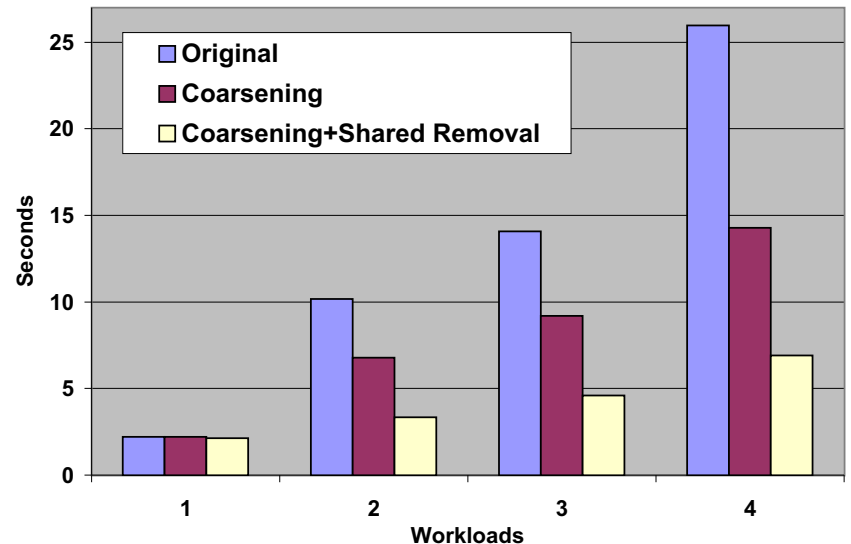

Figure 10: SMG: Time for different Workloads 
increasing simulation overhead. This limits the size of the programs and workloads that they can run. In contrast, ccSIM is an event-based simulator which simulates only memory hierarchies. Our instrumentation tool is flexible and allows us to collect partial traces of only the pertinent memory access. Thus, we can handle a much larger range of programs and workloads. More importantly, these simulators provide only bulk statistics intended for evaluating architecture mechanisms. In contrast, we aim at providing the application programmer with information on the shared-memory behavior of the program and correlate metrics to higher levels of abstraction, such as line numbers and source code data structures.

Execution-driven simulators are a popular approach for implementing memory access simulators. Code annotation tools annotate memory access points. Annotations call handlers, which invoke the memory access simulator. Augmint [25], Proteus [2] and Tango [8] are examples of this approach. All these tools use static code annotation, i.e., they annotate the target code at the source, assembly or object code level. MemSpy [23] and CProf [19] are cache profilers that aim at detecting memory bottlenecks. CProf relies on post link-time binary editing through EEL $[17,18]$. Lebeck and Wood also applied binary editing to substitute instructions that reference data in memory with function calls to simulate caches on-the-fly [20]. Other approaches rely on hardware support, such as watchdogs [6] or statistical sampling with hardware support in ProfileMe [9], to gather information on data references. Scal-Tool detects and quantifies scalability bottlenecks in distributed shared memory architectures, such as the SGI Origin 2000 [29]. It determines inefficiencies due to cache capacity constraints, load imbalance and synchronization. Nikolopoulos et al. discuss OpenMP optimizations for irregular codes based on memory reference tracing to indicate when page migration and loop redistribution is beneficial. This results in comparable performance of optimized OpenMP with MPI parallelization, again on the Origin 2000 [26].

None of the above tools allow misses to be related to source code and data structures. Furthermore, our work differs in the fundamental approach of rewriting binaries, which is neither restricted to a special compiler or programming language, nor does it preclude the analysis of library routines. In addition, execution-driven simulators are often tied to one architecture due to the requirements of annotating the code at assembly or object level. DynInst is available on a number of architectures. Porting our framework to these platforms only involves changing the memory instructions to be instrumented. Another major difference addresses the overhead of large data traces inherent to all these approaches. We allow the analysis of partial traces and employ trace compression to provide compact representations.

SM-prof is an aggregate classification tool for shared-memory references resulting in coherence traffic [3]. It classifies all accesses into access classes depending on how many processors $\mathrm{read} / \mathrm{write}$ to the same data block in the current time slot. It is up to the analyst to find and quantify the location and magnitude of the coherence bottleneck. The analysis tool does not provide this information at the level of individual access points, but only at the level of each access class. This causes to authors "to suspect false sharing" [3]. In contrast, ccSIM is a per-reference coherence analysis tool. We generate detailed coherence statistics for each access point, as well as for global data structures. Metrics include the magnitude of coherence misses, true and false sharing invalidations and classification of invalidations across and in a parallel OpenMP regions. Thus, we do not suspect, we know when false/true sharing occurs (among other symptoms).

The SIGMA (Simulator Infrastructure to Guide Memory Analysis) [10] system has many similarities with our work. It uses post- link binary instrumentation and online trace compression, and allows tagging of metrics to source code constructs. A toolkit by Marin and Mellor-Crummey uses statistical methods based on dynamic measurements of edge counters and histograms of reuse distances for each memory reference to predict cache and execution behavior across different architectural platforms [22]. Both of these approaches are limited to uniprocessor systems while we focus on analyzing coherence traffic for SMPs. The latter work does not focus on transformations, unlike our work.

Recently, most architectures have added hardware counters, which provide information on the frequency of hardware events, e.g., to count shared memory events. Portable APIs like PAPI provide a reasonably platform-independent method of accessing these counters [4]. Hardware counters impose no runtime overhead, and querying counters is typically of low overhead. However, they only provide aggregate statistics without any relation to the source code, and there are only a limited number of counters available. In addition, there are often restrictions on the type of events that can be counted simultaneously. HPCToolkit uses statistical sampling of performance counter data and allows information to be correlated to the program source [24]. Our method goes beyond this granularity by identifying evictors within caches and coherence traffic in SMP to indicate source of inefficiency. A number of commercial tools, such as Intel's VTune, SGI's Speedshop, Sun's Workshop tools also use statistical sampling with source correlation, albeit at a coarser level that HPCToolkit or our approach. It is possible to finer-grained information with customized hardware. The FlashPoint system uses a custom system node controller to monitor coherence events [12]. In general, hardware monitors are fast but may constrain the number of events that can be monitored. At this point in time, they lack a wide acceptance in practice.

Krishnamurthy and Yelick develop compiler analysis and optimization techniques for the shared-memory programming paradigm using SplitC as an example [16]. Their main concern is the hardware-supported coherence model, namely weak consistency. They are specifically concerned about writes and invalidations occurring out-of-order. Their optimizations reflect the constraints of reordering writes in the presence of locks and barriers with respect to weak consistency and employ message pipelining (aggregation of writes) and reduction of communication (two-way to one way or elimination). Satoh et al. study compiler optimizations for OpenMP in a distributed shared memory system based on dataflow techniques to analyze thread interactions [28]. Optimizations include barrier removal and data privatization to reduce coherence-induced messages. Our work shares the aim at optimizing shared-memory applications with these approaches. However, we take a radically different approach by analyzing traces to determine if and where inefficiencies in terms of coherence traffic exist and if there is room for improvements.

\section{Conclusion}

In this paper, we introduced ccSIM, a cache-coherent memory simulator fed by data traces obtained via on-the-fly dynamic binary rewriting of OpenMP benchmarks executing on a Power3 SMP node. We explored the degrees of freedom in interleaving data traces from the different processors with respect to simulation accuracy compared to hardware performance counters. We also provided detailed coherence information per data reference and relate them to their data structures and reference locations in the code.

The experiments conducted by us show close matches of hardware performance counters for coherence events. Furthermore, we demonstrated the feasibility and the benefits of deriving detailed coherence information indicating the location of invalidations in the application code. This detailed level of information allowed us 
to infer opportunities for optimizations that, without ccSIM, could not easily be obtained and localized. This led to program transformations resulting in both significantly decreased coherence traffic and execution time savings. In the future, we intend to assess the benefits of our tool for long-running general-purpose server applications with multithreading on SMPs.

\section{REFERENCES}

[1] Asci purple codes. http://www.llnl.gov/asci/purple, 2002.

[2] E. A. Brewer, C. N. Dellarocas, A. Colbrook, and W. E. Weihl. Proteus: A high-performance parallel-architecture simulator. In Proceedings of the SIGMETRICS and PERFORMANCE'92 International Conference on Measurement and Modeling of Computer Systems, pages 247-248, New York, NY, USA, June 1992. ACM Press.

[3] M. Brorsson. A tool to visualise and find cache coherence performance bottlenecks in multiprocessor programs. In ACM SIGMETRICS Conference, pages 178-187, May 1995.

[4] S. Browne, J. Dongarra, N. Garner, K. London, , and P. Mucci. A scalable cross-platform infrastructure for application performance tuning using hardware counters. In Supercomputing, Nov. 2000.

[5] B. Buck and J. K. Hollingsworth. An API for runtime code patching. The International Journal of High Performance Computing Applications, 14(4):317-329, Winter 2000.

[6] B. R. Buck and J. K. Hollingsworth. Using hardware performance monitors to isolate memory bottlenecks. In ACM, editor, Supercomputing, pages 64-65, 2000.

[7] D. Burger, T. M. Austin, and S. Bennett. Evaluating future microprocessors: The simplescalar tool set. Technical Report CS-TR-1996-1308, University of Wisconsin, Madison, July 1996.

[8] H. Davis, S. R. Goldschmidt, and J. Hennessy. Multiprocessor simulation and tracing using tango. In Proceedings of the 1991 International Conference on Parallel Processing, volume II, Software, pages II-99-II-107, Boca Raton, FL, Aug. 1991. CRC Press.

[9] J. Dean, J. Hicks, C. Waldspurger, W. Weihl, and G. Chrysos. ProfileMe: Hardware support for instruction-level profiling on out-of-order processors. In Proc. 30th Annual IEEE/ACM Int. Symp. on Microarchitecture (MICRO-97), pages 292-302, Dec. 1997.

[10] L. DeRose, K. Ekanadham, J. K. Hollingsworth, , and S. Sbaraglia. SIGMA: A simulator infrastructure to guide memory analysis. In Supercomputing, Nov. 2002.

[11] R. D. F. E. Chow, A. J. Cleary. Design of the hypre preconditioner library. In SIAM Workshop on Object Oriented Methods for Inter-operable Scientific and Engineering Computing, Oct. 1998.

[12] J. Gibson. Memory Profiling on Shared Memory Multiprocessors. PhD thesis, Stanford University, July 2003.

[13] W. Gunsteren and H. Berendsen. Gromos: Groningen molecular simulation software. Tr, Laboratory of Physical Chemistry, University of Groningen, 1988.

[14] C. Hughes, V. Pai, P. Ranganathan, and S. Adve. Rsim: Simulating Shared-Memory Multiprocessors with ILP Processors. IEEE Computer, 35(2):40-49, February 2002.

[15] H. Jin, M. Frumkin, and J. Yan. The openmp implementations of nas parallel benchmarks and its performance. TR NAS-99-011, NASA Ames Research Center, Oct. 1999.

[16] A. Krishnamurthy and K. Yelick. Optimizing parallel programs with explicit synchronization. In ACM SIGPLAN Conference on Programming Language Design and Implementation, pages 196-204, 1995.

[17] J. Larus and T. Ball. Rewriting executable files to measure program behavior. Software Practice \& Experience, 24(2):197-218, Feb. 1994.

[18] J. R. Larus and E. Schnarr. EEL: Machine-independent executable editing. In ACM SIGPLAN Conference on Programming Language Design and Implementation, pages 291-300, June 1995.

[19] A. R. Lebeck and D. A. Wood. Cache profiling and the SPEC benchmarks: A case study. Computer, 27(10):15-26, Oct. 1994.

[20] A. R. Lebeck and D. A. Wood. Active memory: A new abstraction for memory system simulation. ACM Transactions on Modeling and Computer Simulation, 7(1):42-77, Jan. 1997.

[21] J. Marathe, F. Mueller, T. Mohan, B. R. de Supinski, S. A. McKee, and A. Yoo. Metric: Tracking down inefficiencies in the memory hierarchy via binary rewriting. In International Symposium on Code Generation and Optimization, pages 289-300, Mar. 2003.

[22] G. Marin and J. Mellor-Crummey. Cross architecture performance predictions for scientific applications using parameterized models. In SIGMETRICS Conference on Measurement and Modeling of Computer Systems, page (to appear), 2004.

[23] M. Martonosi, A. Gupta, and T. Anderson. Memspy: analyzing memory system bottlenecks in programs. In Proceedings of the 1992 ACM SIGMETRICS joint international conference on Measurement and modeling of computer systems, pages 1-12, 1992.

[24] J. Mellor-Crummey, R. Fowler, and D. Whalley. Tools for application-oriented performance tuning. In International Conference on Supercomputing, pages 154-165, June 2001.

[25] A.-T. Nguyen, M. Michael, A. Sharma, and J. Torrellas. The augmint multiprocessor simulation toolkit: Implementation, experimentation and tracing facilities. In IEEE International Conference on Computer Design: VLSI in Computers and Processors, pages 486-491, Washington - Brussels - Tokyo, Oct. 1996. IEEE Computer Society.

[26] D. Nikolopoulos, C. Polychronopoulos, and E. Ayguade. Scaling irregular parallel codes with minimal programming effort. In Supercomputing, 2001.

[27] M. Rosenblum, S. A. Herrod, E. Witchel, and A. Gupta. Complete computer system simulation: The SimOS approach. IEEE parallel and distributed technology: systems and applications, 3(4):34-43, Winter 1995.

[28] S. Satoh, K. Kusano, and M. Sato. Compiler optimization techniques for openMP programs. Scientific Programming, 9(2-3):131-142, 2001.

[29] Y. Solihin, V. Lam, and J. Torrellas. Scal-tool: Pinpointing and quantifying scalability bottlenecks in dsm multiprocessors. In Supercomputing, Nov 1999.

[30] J. Vetter and F. Mueller. Communication characteristics of large-scale scientific applications for contemporary cluster architectures. In International Parallel and Distributed Processing Symposium, Apr. 2002.

[31] J. Vetter and F. Mueller. Communication characteristics of large-scale scientific applications for contemporary cluster architectures. Journal of Parallel Distributed Computing, 63(9):853-865, Sept. 2003. 